\title{
Strategies for creating a collaborative network in research and teaching: CEDACORE
}

$\mathrm{B}$ etween 14 and 18 October 2017 six leading researchers and 33 bright and enthusiastic early career researchers in the fields of paediatric dentistry, restorative dentistry and psychology from across Brazil and the UK met in the city of Goiânia, Midwest Brazil to create a collaboration for enhancing the care and cure of children with dental caries across the world.

The global burden of oral diseases is attracting public attention and causing political debate. Young children and their families are still suffering the negative impacts of dental caries. Dental treatment is still depicted as anxiety-provoking. Thankfully, the scientific evidence on the efficiency of minimum intervention oral healthcare is getting stronger. When a unique opportunity to fund a high level scientific meeting arose, early career researchers and leading researchers from the UK and Brazil gathered in the heart of Brazil and launched CEDACORE: Children Experiencing Dental Anxiety: Collaboration On Research and Education.

\section{Antecedents: planning the workshop}

The Newton Fund Researcher Links Workshops called for applicants in April 2016, aiming to support meetings with direct relevance to partners' countries' welfare and economic development. Proposals had to 'support international development-relevant research, contribute to capacity building of early career researchers and establish new research links or significantly develop existing links, and have the potential for longer term sustainability'. Brazil was one of the countries invited to apply for the funding.

Dental caries is the commonest disease of children in the world today (WHO). Around 53\% of the 20 million Brazilian children aged five-years-old have dental caries and more than $80 \%$ have not received treatment for it (National Survey, 2010) and suffer the negative impact of dental caries, especially pain and lowered oral health-related quality of life. One of the reasons for the lack of treatment provision is because many children find it hard to co-operate with treatment in the dental chair, due to behaviour problems or dental anxiety. In Brazil, the majority of them are from low-income families and do not have access to private dental treatment. Even though Brazil has one of the best public health services in the world ('Sistema Unico de Saude' - SUS), it does not provide paediatric dental treatment under sedation or general anaesthesia as a rule. Therefore, Brazilian dentists usually do not receive formal training on children's sedation. On the other hand, the UK has national standards and guidelines for dentists to provide conscious sedation. Brazil and the UK have also begun to adopt less invasive approaches to restoring teeth, and these might make it easier for anxious children to accept.

Considering that the dental caries fits the ODA concept (Official development assistance [ODA] is defined as government aid designed to promote the economic development and welfare of developing countries [available at OECD.org]) as required by the Newton Fund notice, Professor Marie Therese Hosey (King's College London) and Professor Luciane R. R. S. Costa (Universidade Federal de Goiás, Brazil) put together a proposal aiming to: share the knowledge on child behaviour management, sedation and minimally invasive filling techniques; advance the partnership that has already been started between the two academic institutions; establish new research networks between the two countries and among participants; and to propose a course plan to be taught in different Brazilian graduate programmes that addresses the behavioural management of challenging children.

The proposal was submitted in June 2016, was successful and accepted for a funding from the Newton Fund of $£ 42,000$ in November 2016. The 'International Research Collaboration for Managing Anxious Children who Need Dental Treatment' was one of eight approved Brazilian proposals.

Table 1 The lecture programme

Themes

- The 'care and cure' of challenging children - setting the agenda for the workshop: What would the perfect service look like?

- The psychologist's perspective on the challenging child attending the dental setting. What is cognitive behavioural therapy (CBT)?

- Contemporary Minimum Intervention (MI) non-operative preventive caries management. Consensus recommendations on minimally invasive operative caries management.

- An overview of Brazilian practices for managing challenging children undergoing dental treatment; the NESO (Universidade

- A panorama of Brazil's contribution for managing anxious children who need dental treatment: teaching and research in postgraduate programmes.

Faculty

Professors in King's College London:

- Marie Therese Hosey: Head of Paediatric Dentistry at King's College London; member of the International Committee for the Advancement of Procedural Sedation (ICAPS)

- Avijit Banerjee: Head in Cariology \& Operative Dentistry and Honorary Consultant and Clinical Lead in Restorative Dentistry at King's College London

- Tim Newton: Head of Psychology as Applied to Dentistry, Honorary Consultant Health Psychologist at King's College London.

Professors in the Universidade Federal de Goiás and members of the ICAPS:

- Luciane R. R. S. Costa: Paediatric Dentist, Coordinator of the dental sedation centre 'NESO'

- Paulo S. S. Costa: Paediatrician, Coordinator of the Graduate Programme in Health Sciences

- Professor in the University of São Paulo

- Marcelo J. S. Bönecker: Head of the Paediatric Dentistry department, President-elect of the International Association of Paediatric Dentistry. 


\section{The undertaking}

Coordinators and mentors gave dynamic short lectures in an interactive atmosphere (Table 1). In addition, participants presented press releases of their research and were grouped to: plan a specialist course for the management of challenging children undergoing dental treatment; and to establish innovative and relevant research questions to be developed in collaborative projects in the short and medium term.

\section{It's time to nurture CEDACORE}

The workshop has provided opportunities for early career researchers to interact, learn from each other and explore opportunities for building long-lasting research collaborations. Participants' conclusions were:

- Children with anxiety or behaviour management problems can be managed by trained dentists integrated in collaboration with medical specialties, including psychology

- The concepts of 'having knowledge of', 'experience in' and 'competence in' in a postgraduate curriculum must be applied during the dentists' training for managing challenging children

- Research themes to be explored in future projects are: minimally invasive dentistry, pharmacological management of anxious children, cognitive behavioural therapy and challenging behaviour.

All in all, the exchange of ideas, values and culture between UK and Brazil has led to a promising future in which children will have a chance of better care and cure of their oral health. The workshop has underpinned the CEDACORE.

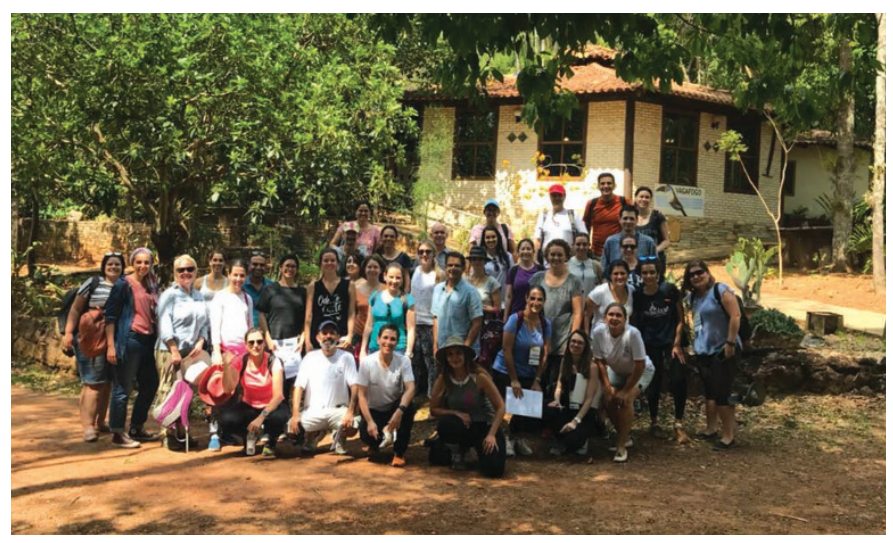

Participants: Aline de Almeida Neves, Adriana de Sales Cunha Correia, Andrea Vaz Braga Pintor, Anelise Daher Vaz Castro, Carla Massignan, Carolina Dea Bruzamolin, Cristiane Baccin Bendo, Geovanna de Castro Morais Machado, Heloisa de Sousa Gomes Rodrigues, Karoline Alves Viana, Lívia Fulgêncio Libânio, Lucas Guimarães Abreu, Mariana Passos de Luca, Marília Leão Goettems, Marina Sousa Azevedo, Patrícia Corrêa de Faria, Paulo Veríssimo Barbosa D’Almeida, Regina Mota de Carvalho, Renata Andrea Salvitti de Sá Rocha, Taís de Souza Barbosa, Tatiana Kelly da Silva Fidalgo, Vanessa Polina Pereira da Costa, Alaa Hani Bani, Chrysoula Tatsi, Ellie Heidari, Geanina Bruj, Jennifer Hare, Makbule Sipahi Ogretme, Malihe Moeinian, Mike Harrison, Mona Agel, Sanjeev Sood. We would like to acknowledge Mona Agel for drafting this report.

\section{Strategy and initiatives launch event held in Greater Manchester}

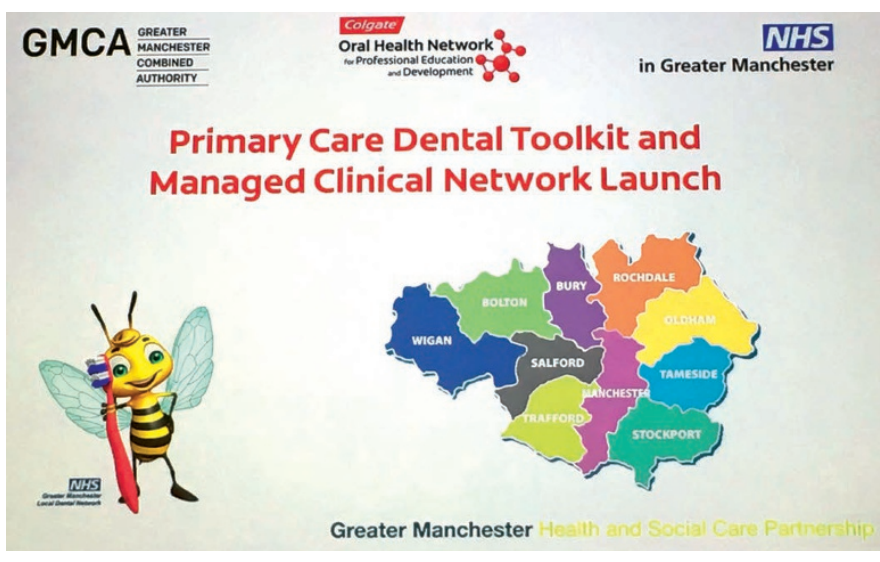

On 7 December the Local Professional Dental Network (LDN), as part of Greater Manchester Health \& Social Care Partnership, held a launch event to update practitioners on the dental commissioning strategy and initiatives across the Greater Manchester region. The event was supported by Colgate and attended by over 200 local practitioners, DCPs and management teams.

The first part of the event gave managed clinical network (MCN) chairs an opportunity to discuss the concepts, development and future of the networks. There are now six MCNs operating across Greater Manchester, representing services and pathway delivery for restorative, orthodontic, oral surgery, special care, paediatric dentistry and oral health improvement services. The session was concluded with a summary of the activity of the Dental Advisory Group, which provides primary dental care representation and engagement within the devolved Greater Manchester system. Following this, the group held a market engagement event and supper which gave local practitioners a chance to ask questions and engage with teams on key strategies across local GM services, such as the Pride in Practice programme working with the LGBT Foundation.

The second session focussed on the development programme of work by the LDN presenting toolkits that have been conceived and developed by local practitioners to support best clinical practice and quality for patients. These included the Saving Smiles Trauma Toolkit, Medical Histories Do Matter, the Early Years \& Baby Teeth Do Matter, and the Healthy Living Dentistry Programme. The LDN is very keen to share with others and has locally adopted work by colleagues in Cheshire \& Merseyside of the Dementia Friendly and Older People Toolkit. All of these toolkits will be given to practices in printed and electronic form. These quick reference guides can be stored on computer desktops for rapid access and help dentists navigate some of the more challenging aspects of primary care practice.

Chair of the LDN Mohsan Ahmad said: 'The event gave our LDN the opportunity to showcase some of the excellent programmes that have been developed for primary care dental teams. The attendees also heard about how our MCNs are crossing barriers between primary and secondary care to work together to improve the services offered to our patients'.

As these initiatives evolve and the work of the GM LDN continues, we look forward to further engagement events and opportunities in the future. Further information is available from england.gmdental@nhs.net.

By James Darcey 